\title{
Aciclovir no tratamento da varicela em idade pediátrica: revisão baseada na evidência
}

Tânia Colaço,* Maria Espírito Santo*

\section{RESUMO}

Objectivos: A varicela é uma doença benigna que afecta sobretudo as crianças, sendo a taxa de incidência em Portugal (2005) de 2,37\%. Apesar de auto-limitada, é altamente contagiosa e podem ocorrer complicações em 5-10\% de todos os indivíduos afectados. O objectivo deste trabalho foi rever a evidência existente sobre os benefícios da utilização do aciclovir, no tratamento da varicela na população pediátrica imunocompetente, em relação à sintomatologia, duração da doença e complicações associadas. Fontes de dados: Medline, sítios de medicina baseada na evidência, Índex de Revistas Médicas Portuguesas e referências bibliográficas dos artigos seleccionados.

Métodos de revisão: Pesquisa de revisões sistemáticas (RS), meta-análises (MA), ensaios clínicos aleatorizados e controlados (ECAC) e normas de orientação clínica (NOC), utilizando as palavras-chave: chickenpox e acyclovir. Foi utilizada a taxonomia SORT para apresentação dos resultados.

Resultados: Foram encontrados 105 artigos, dos quais foram seleccionados 11: duas RS, três ECAC e seis NOC. Da análise das RS e ECAC há a referir que o uso de aciclovir oral na varicela está associado à redução do número de dias de febre e do número máximo de lesões. Estes efeitos são modestos e verificam-se quando o fármaco é administrado nas primeiras 24 horas de exantema. Não existem diferenças clinicamente importantes entre o aciclovir e o placebo relativamente a complicações secundárias à infecção. Assim sendo, o uso de aciclovir oral não está recomendado, por rotina, em imunocompetentes com varicela em idade pediátrica (Recomendação A). As NOC são consistentes com esta recomendação.

Conclusões: Para além do efeito marginal do aciclovir na melhoria dos sintomas, a necessidade de administração precoce do fármaco bem como a sua posologia dificultam a sua utilização. São, ainda, necessários estudos de custo-benefício relativos ao aciclovir e estudos de segurança e eficácia de outros antivíricos na população pediátrica.

Palavras-chave: Aciclovir; Varicela; População Pediátrica.

\section{INTRODUÇÃO}

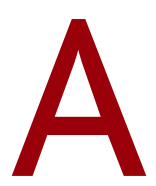
varicela é uma doença vírica provocada pelo vírus varicela-zoster que afecta, sobretudo, crianças. É benigna e auto-limitada, mas altamente contagiosa. ${ }^{1}$ As complicações surgem em $5-10 \%$ dos indivíduos afectados, sendo raras em imunocompetentes e mais comuns em recém--nascidos, adultos e imunocomprometidos. ${ }^{2}$ A taxa de incidência estimada para Portugal em 2005, com base nos dados dos Médicos Sentinela, foi de 2,37\%. ${ }^{3}$

O tratamento desta patologia pode ser sintomático (alívio da febre e do prurido) ou orientado directamen-

*Interna de Medicina Geral e Familiar, Unidade de Saúde Familiar Horizonte, Centro de Saúde de Matosinhos. te para a etiologia da doença, com imunoglobulinas e antivíricos. O aciclovir é um dos antivíricos disponíveis no mercado que previne a replicação do vírus varicela-zoster tendo, por isso, o potencial de melhorar a sintomatologia numa fase mais precoce da doença. Esta acção é obtida com efeitos adversos mínimos.

Tratando-se de uma doença de curso benigno e auto-limitado é discutível a utilização de um tratamento etiológico. Contudo, o facto de apresentar uma taxa de incidência considerável confronta o Médico de Família, durante a sua prática clínica, com a pergunta: o uso de aciclovir é benéfico na redução da gravidade e da duração da varicela (não complicada) em crianças imunocompetentes?

O objectivo deste estudo foi rever a evidência exis- 
tente sobre o benefício do uso de aciclovir na varicela em crianças imunocompetentes, no que se refere ao alívio dos sintomas, duração da doença e complicações da mesma.

\section{MÉTODOS}

Foi efectuada uma pesquisa nas bases de dados UpToDate, Cochrane, DARE, Bandolier, TRIP Database, National Guideline Clearinghouse, Guidelines Finder, Canadian Medical Association Practice Guidelines Infobase, Medline, Índex de Revistas Médicas Portuguesas, de Normas de Orientação Clínica (NOC), revisões sistemáticas e de artigos originais, publicados até Setembro de 2008. Consultaram-se, ainda, as referências bibliográficas dos artigos seleccionados.

A pesquisa foi realizada utilizando como palavraschave chickenpox e acyclovir (termos MESH), para os sítios de língua inglesa, e varicela e aciclovir, para o Índex de Revistas Médicas Portuguesas. Foram incluídos estudos de meta-análise, revisões sistemáticas, ensaios clínicos aleatorizados e controlados (ECAC) e NOC, escritos em língua portuguesa, inglesa, espanhola ou francesa. Seleccionaram-se os artigos correspondentes à população imunocompetente em idade pediátrica (0 - 18 anos) e nos quais a intervenção (uso de aciclovir) fosse comparada com o uso de placebo.

A classificação desta revisão foi baseada na Taxonomia SORT. ${ }^{4}$

\section{RESULTADOS}

Encontraram-se 105 artigos, de entre os quais foram seleccionados 11: duas revisões sistemáticas, três ECAC e seis NOC. Os restantes foram excluídos por discordância com o objectivo, não cumprimento dos critérios de inclusão ou por se encontrarem repetidos.

Os três ECAC ${ }^{5-7}$ encontrados (Quadro I) referem-se a estudos efectuados no início da década de noventa e a todos eles foi atribuído o nível de evidência 2. Em todos estes estudos era comparado o efeito do uso de aciclovir iniciado nas primeiras 24 horas de doença com o uso de placebo.

As variáveis estudadas foram o número máximo de lesões de varicela e o número de dias decorridos até: número máximo de lesões, ausência de novas lesões, ausência de febre e redução de prurido. Avaliaram-se, ainda, as complicações associadas à doença e os efeitos secundários relacionados com o uso do fármaco.

Os resultados encontrados nos três ECAC, relativamente às diferentes variáveis estudadas, estão discriminados no Quadro I. Apesar de se encontrarem várias diferenças estatisticamente significativas nos três estudos, apenas a redução do número de dias até à ausência de febre se revelou concordante em todos eles. No que diz respeito às complicações e efeitos secundários nenhum dos três ensaios encontrou diferença clinicamente significativa entre os grupos em estudo.

A pesquisa efectuada resultou, também, na identificação de duas revisões sistemáticas (Quadro II), às quais se atribuiu um nível de evidência 1. A primeira, uma revisão da Cochrane, ${ }^{2}$ incluiu os três ECAC já referidos. A amostra continha um total de 988 indivíduos dos 2 aos 18 anos. Apenas se encontrou diferença esta-

\section{QUADRO I. Ensaios clínicos aleatorizados e controlados}

\begin{tabular}{|c|c|c|c|c|c|c|c|c|}
\hline \multirow[b]{2}{*}{ Autor } & \multirow[b]{2}{*}{$\begin{array}{l}\text { Nível } \\
\text { evid }\end{array}$} & \multirow[b]{2}{*}{$\mathbf{n}$} & \multirow[b]{2}{*}{$\begin{array}{l}\text { Nº máx } \\
\text { lesões }\end{array}$} & \multicolumn{3}{|c|}{ Dias até } & \multirow[b]{2}{*}{$\begin{array}{c}\downarrow \\
\text { prurido }\end{array}$} & \multirow[b]{2}{*}{$\begin{array}{c}\text { Compl e efeitos } \\
\text { secundários }\end{array}$} \\
\hline & & & & $\begin{array}{l}N^{\circ} \text { máx } \\
\text { lesões }\end{array}$ & $\begin{array}{c}\varnothing \text { novas } \\
\text { lesões }\end{array}$ & $\begin{array}{c}\varnothing \\
\text { febre }\end{array}$ & & \\
\hline Balfour et al, 1990 & 2 & $\begin{array}{c}105 \\
\text { (5-16 anos) }\end{array}$ & $\downarrow *$ & $\downarrow *$ & $\downarrow$ & $\downarrow *$ & $\downarrow$ & $\varnothing$ diferença \\
\hline Dunkle et al, 1991 & 2 & $\begin{array}{c}815 \\
\text { (2-12 anos) }\end{array}$ & $\downarrow *$ & NA & $\downarrow *$ & $\downarrow *$ & $\downarrow *$ & $\varnothing$ diferença \\
\hline Balfour et al, 1992 & 2 & $\begin{array}{c}68 \\
(13-18) \text { anos }\end{array}$ & $\downarrow$ & $\downarrow *$ & $\downarrow *$ & $\downarrow *$ & NA & $\varnothing$ diferença \\
\hline
\end{tabular}

n: tamanho amostral (grupo etário); Nível evid: nível de evidência; máx: máximo; $\downarrow$ : diminuição; *: estatisticamente significativo; Compl: complicações; Ø: ausência; NA: não aplicável. 


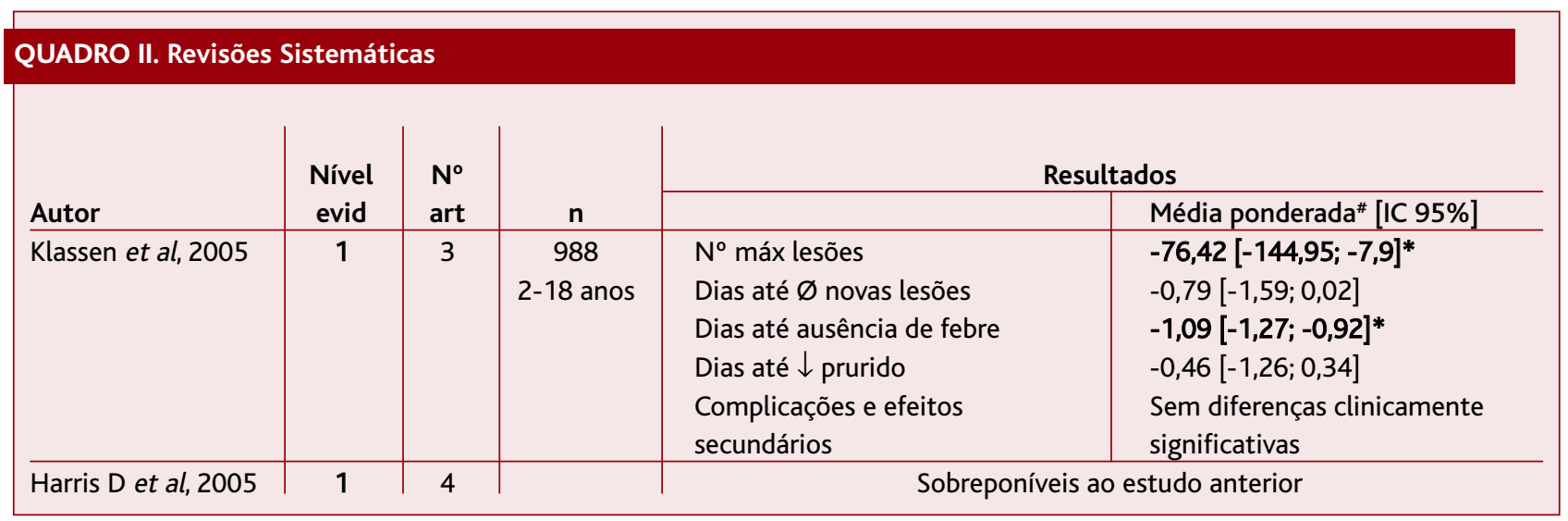

Nível evid: nível de evidência; $N^{\circ}$ art: número de artigos; $n$ : tamanho amostral (grupo etário); \#: média ponderada da diferença de número de lesões ou número de dias; IC: intervalo de confiança; máx: máximo; $\downarrow$ : diminuição; *: estatisticamente significativo; $\varnothing$ : ausência.

tisticamente significativa em relação ao número máximo de lesões e aos dias até ausência de febre, ou seja, o grupo de indivíduos a fazer aciclovir teve, em média, menos 76 lesões e menos um dia de febre que o grupo ao qual foi administrado placebo. Nesta revisão não houve diferença clinicamente significativa entre os grupos, no que se refere ao aparecimento de complicações da doença ou efeitos secundários do fármaco.

A segunda revisão $0^{8}$ encontrada apresenta resultados sobreponíveis aos descritos no parágrafo anterior, uma vez que inclui, além dos três ECAC do Quadro I, a revisão da Cochrane (Quadro II).

Como já foi referido, encontraram-se seis NOC, ${ }^{1,9-11}$ todas elas reconhecendo um efeito terapêutico modesto ao uso de aciclovir oral no tratamento da varicela não complicada, na população pediátrica imunocompetente. Em nenhuma NOC é recomendado o uso por rotina deste fármaco. As normas referem, ainda, que $o$ uso de aciclovir oral em indivíduos infectados por cohabitante é controverso e que será de ponderar o uso de aciclovir oral em indivíduos com idade $\geq 13$ anos (nível de evidência 3).

$\mathrm{Na}$ análise destes resultados é necessário considerar alguns aspectos importantes. Como se pode ver no Quadro I, os estudos Balfour $1990^{5}$ e Balfour $1992^{6}$ têm amostras de pequena dimensão e os três ensaios referem-se a grupos etários diferentes. Apesar de em todos eles se encontrarem várias diferenças estatisticamente significativas, estas foram consensuais em apenas uma das variáveis. O facto de os grupos etários estudados não serem sobreponíveis nos três ECAC pode ter tido influência nestes resultados.

O ensaio Balfour 1990 faz uma discussão inadequada sobre os casos de abandono do estudo. Os estudos de Dunkle $1991^{7}$ e Balfour 1992 são, no fundo, dois subestudos utilizando a mesma metodologia para diferentes faixas etárias e, em ambos, a descrição dos métodos de aleatorização e dupla ocultação surge pouco desenvolvida. É necessário referir, ainda, que todos estes ECAC tiveram financiamento da indústria farmacêutica.

Na revisão da Cochrane ${ }^{2}$ não foi realizada meta-análise devido ao pequeno número de estudos e às diferentes populações em estudo.

Com base nos dados até aqui expostos, pode dizer-se que o uso de aciclovir oral diminui, em um dia, a duração de febre e diminui o número máximo de lesões (nível de evidência 1). É importante realçar que estes efeitos foram encontrados com a administração do fármaco nas primeiras 24 horas de exantema. Há a acrescentar que o uso de aciclovir oral não afecta de forma clinicamente significativa a taxa de complicações secundárias da infecção (nível de evidência 1).

Considerando todos os resultados descritos, pode-se dizer que o uso de aciclovir oral não está recomendado em indivíduos imunocompetentes com varicela em idade pediátrica (força de recomendação A). A utilização deste fármaco será de ponderar em indivíduos com idade $\geq 13$ anos ou infectados por co-habitante (força de recomendação C). 


\section{CONCLUSÕES}

O efeito do aciclovir no tratamento da varicela é marginal, numa doença que é benigna e auto-limitada. Acresce, ainda, que os efeitos descritos para o uso deste fármaco se verificam quando este é iniciado nas primeiras 24 horas de exantema, uma janela diagnóstica difícil de alcançar.

Considerando os resultados encontrados nesta revisão, pode concluir-se que o uso de aciclovir oral não está recomendado em indivíduos imunocompetentes com varicela em idade pediátrica (força de recomendação A). A utilização deste fármaco será de ponderar em indivíduos com idade $\geq 13$ anos ou infectados por cohabitante (força de recomendação C).

O uso do aciclovir poderá colocar algumas dificuldades de adesão, uma vez que tem de ser administrado em quatro tomas diárias durante cinco dias. ${ }^{12}$

Existem outros anti-víricos no mercado (fanciclovir e valganciclovir) com maior biodisponibilidade, mas para os quais não existem estudos em idade pediátrica, desconhecendo-se, por isso, o efeito que podem ter no tratamento da varicela em crianças imunocompetentes.

Neste momento, não existem dados sobre os efeitos do uso sistemático do fármaco. É, também, desconhecida a análise de custo-benefício que considere o custo do fármaco, o custo acrescido de consultas (para diagnóstico da doença e prescrição do medicamento) e o custo do absentismo, quer escolar (do indivíduo afectado), quer laboral (do cuidador do indivíduo). Estima-se que este último seja o factor mais implicado nos custos relacionados com a varicela, sendo necessários estudos que forneçam dados mais concretos para ponderação do custo-benefício da utilização de aciclovir.

\section{REFERÊNCIAS BIBLIOGRÁFICAS}

1. Albrecht MA. Treatment and prevention of chickenpox. UpToDate. Disponível em: http://www.uptodateonline.com [acedido em 26/05/2008].

2. Klassen TP, Belseck EM, Wiebe N, Hartling L. Acyclovir for treating varicella in otherwise healthy children and adolescents. Cochrane Database Syst Rev 2004; (2): CD002980.
3. Médicos Sentinela. Relatório das actividades de 2005. Lisboa: Instituto Nacional de Saúde Dr. Ricardo Jorge; 2007.

4. Ebell MH, Sikew J, Weiss BD, Woolf SH, Susman J, Ewigman B, et al. Strength of Recommendation Taxonomy (SORT): a patient-centered approach to grading evidence in the medical literature. Am Fam Phys 2004 Feb 1; 69 (3): 548-56.

5. Balfour HH Jr, Kelly JM, Suarez CS, Heussner RC, Englund JA, Crane DD, et al. Acyclovir treatment of varicella in otherwise healthy children. J Pediatr 1990 Apr; 116 (4): 633-9.

6. Balfour HH Jr, Rotbart HA, Feldman S, Dunkle LM, Feder HM Jr, Prober CG, et al; The Collaborative Acyclovir Varicella Study Group. Acyclovir treatment of varicella in otherwise healthy adolescents. J Pediatr 1992 Apr; 120 (4): 627-33.

7. Dunkle LM, Arvin AM, Whitley RJ, Rotbart HA, Feder HM Jr, Feldman S, et al. A controlled trial of acyclovir for chickenpox in normal children. N Engl J Med 1991 Nov 28; 325 (22): 1539-44.

8. Harris D, Redhead J. Should acyclovir be prescribed for immunocompetent children presenting with chickenpox? Arch Dis Child 2005 Jun; 90 (6): 648-50.

9. American Academy of Pediatrics Committee on Infectious Diseases. The use of oral acyclovir in otherwise healthy children with varicella. Pediatrics 1993 Mar; 91 (3): 674-6.

10. Ogilvie MM. Antiviral prophylaxis and treatment in chickenpox. A review prepared for the UK Advisory Group on Chickenpox on behalf of the British Society for the Study of Infection. J Infect 1998 Jan; 36 (1): 31-8.

11. Tarlow MJ, Walters S. Chickenpox in childhood. A review prepared for the UK Advisory Group on Chickenpox on behalf of the British Society for the Study of Infection. J Infect 1998 Jan; 36 Suppl 1: 39-47.

12. Anjos R, Bandeira T, Marques JG. Formulário de Pediatria. $3^{\text {a }}$ ed. Lisboa: Esteve Farma; 2004.

Os autores declararam não possuir conflitos de interesses

\author{
ENDEREÇO PARA CORRESPONDÊNCIA \\ Tânia Colaço \\ Rua Dr. Alfredo Cunha, $n^{\circ} 365$ \\ 4450-021 Matosinhos \\ E-mail: tcacolaco@hotmail.com
}

Recebido em 23/12/2008

Aceite para publicação em 13/04/2009 
ABSTRACT

\section{ACYCLOVIR FOR CHICKENPOX TREATMENT IN CHILDREN: EVIDENCE-BASED REVIEW}

Goals: Chickenpox is a benign disease that affects mostly children, showing an incidence rate of $2,37 \%$ in Portugal (2005). Despite the fact that it is a self-limited disease, it is highly contagious and can have complications in $5-10 \%$ of all affected individuals. The aim of this work is to review the existing evidence about the benefits of using acyclovir in chickenpox treatment in the immunocompetent pediatric population, concerning symptoms, days of illness and complications.

Data sources: Medline, evidence-based medicine sites, the Portuguese Medical Magazines Index (RMP) and bibliographic references of the selected articles.

Review methods: Research of systematic reviews (SR), meta-analysis (MA), randomized controlled trials (RCT) and guidelines (G), using the key-words: chickenpox and acyclovir. SORT taxonomy was used to present the results.

Results: 105 articles were found, 11 of which were selected: two SR, three RCT and six G. SR and RCT analysis show that the use of oral acyclovir in chickenpox treatment is associated with a reduction of the number of days of fever and the maximum number of lesions. These are small effects that are only present when the medication is started during the first $24 \mathrm{~h}$ of rash. There are no clinically important differences concerning chickenpox secondary complications between the acyclovir and placebo treatment. Consequently, the use of oral acyclovir is not routinely recommended in the immunocompetent paediatric population (Recommendation A). The guidelines are consistent with this recommendation.

Conclusions: Apart from the modest effects of acyclovir in symptoms improvement, the need for an early administration of this medication, as well as its posology, turns difficult its use. Further studies are needed to evaluate acyclovir cost-benefit relation, and the safety and efficacy of other antiviral drugs in children.

Keywords: Acyclovir; Chickenpox; Paediatric Population. 